\title{
Closed timelike curves in asymmetrically warped brane universes
}

\author{
Heinrich Päs ${ }^{1}$, Sandip Pakvasa ${ }^{2}$, James Dent ${ }^{3}$, Thomas J. Weiler ${ }^{3}$ \\ ${ }^{1}$ Fakutät für Physik, Technische Universität Dortmund, D-44221 Dortmund, Germany \\ 2 Department of Physics 83 Astronomy, University of Hawaii at Manoa, 2505 Correa Road, \\ Honolulu, HI 96822, USA \\ 3 Department of Physics and Astronomy, Vanderbilt University, Nashville, TN 37235, USA
}

December 3, 2013

\begin{abstract}
In asymmetrically warped spacetimes different warp factors are assigned to space and to time. We discuss causality properties of these warped brane universes and argue that scenarios with two extra dimensions may allow for timelike curves which can be closed via paths in the extra-dimensional bulk. In particular, necessary and sufficient conditions on the metric for the existence of closed timelike curves are presented. We find a six-dimensional warped metric which satisfies the CTC conditions, and where the null, weak and dominant energy conditions are satisfied on the brane (although only the former remains satisfied in the bulk). Such scenarios are interesting, since they open the possibility of experimentally testing the chronology protection conjecture by manipulating on our brane initial conditions of gravitons or hypothetical gauge-singlet fermions ("sterile neutrinos") which then propagate in the extra dimensions.
\end{abstract}

\section{Introduction}

The physics of time travel has fascinated science fiction aficionados and scientists alike. In particular, the seminal papers of Morris, Thorne and Yurtsever [1] on traversable wormholes initiated a considerable research library of serious attempts to transmit information to the past, i.e. to generate closed timelike curves (CTCs). Several spacetime settings, mostly contrived or oversimplified in some way, have been discussed in the literature. These include Gödel's rotating universe [2], the rotating cylinder of van Stockum and Tipler [3, 4], Gott's pair of moving cosmic strings [5] 13, Wheeler's spacetime foam [7], regions inside the horizon

\footnotetext{
${ }^{1}$ Compare, however, the arguments given in [6].
} 
of Kerr- and Kerr-Newman geometries [8], Alcubierre's warp drive [9], and Ori's vacuum torus [10]. Typically these spacetimes suffer from obstacles of either unphysically fast rotation to tip the Lorentz cones, or the requirement of exotic matter with negative energy density which violates the so-named null, weak, strong and dominant energy conditions. Several analyses indicate possible instabilities of such spacetimes to classical perturbations and/or quantum fluctuations [11]. This situation has inspired Stephen Hawking's "chronology protection conjecture" [12], which states that the ultimate laws of physics prevent the appearance of CTCs. Hawking's "chronology horizon" [12] is a special type of Cauchy horizon, which separates spacetime regions where CTCs occur from spacetime regions where chronology is protected. Although apparently plausible situations seem naively to violate causality, quantum corrections to the stress-energy tensor diverge in semi-classical calculations at the chronology horizon. It has been argued that the back-reaction to the metric would destroy the potential time-machine on the horizon.

Whether Hawking's chronology protection conjecture holds beyond the semi-classical treatment, so that chronology is truly protected, is still not known. Very probably a better understanding of quantum gravity will be necessary to resolve this issue in the future. In the meantime, a study of physics under the unusual conditions surrounding the chronology horizon may provide more insight into chronology protection. One might glimpse some fascinating new physics proposed to avoid the obvious paradoxes associated with time travel. These paradoxes include the Grandfather and Bootstrap paradoxes. In the Grandfather paradox, one modifies the initial conditions that lead to one's own existence; in the Bootstrap paradox, an effect is its own cause. If the chronology protection conjecture is false, even more wonderful discoveries may await the serious researcher. Proposals include nonHausdorff manifold geometry [13], where the same event has multiple futures or pasts, and the many-world interpretation of quantum mechanics, with switching between parallel histories [14].

The advent of theories with large extra dimensions has provided yet new room for chronology violations (see e.g. the discussion in [15]). Extra dimensions were originally motivated by the consistency of string theory.

More recently, large (or even infinite) extra dimensions have been discussed as a possible new way to understand the hierarchy problem $\left(M_{\text {weak }} \ll M_{P}\right)$ [16, 17] (for reviews see e.g. [18]) and to keep neutrino masses small [19]. In many extra-dimension models, ordinary Standard Model (SM) fields are confined on a brane (our three-surface), while gravitons and other hypothetical SM singlet fields are allowed to propagate also in the extra-dimensional 
bulk. A generic feature of such spacetimes seems to be the existence of signals, mediated by the graviton or SM singlets, taking "shortcuts" through the extra dimension. As viewed from our brane world, these shortcuts appear as superluminal communication [20]. Such apparent superluminal communication, via graviton shortcuts in the bulk [21], or earlier, via wormholes [22], has been proposed as a possible solution to the cosmological horizon problem (relaxing one of the needs for an inflationary epoch in the early universe). While there seems to be agreement in the literature that extra dimensional spacetimes admit bulk shortcuts under rather generic conditions, whether these shortcuts solve the horizon problem depends on the details of the specific extra-dimensional model [23, 24, 25, 26].

In this paper we discuss causality violations arising in a particularly interesting class of extra-dimensional scenarios allowing for bulk shortcuts, so-called asymmetrically warped spacetimes. Asymmetric warping assigns a different warp factor to time versus space coordinates. We work with lightlike world-lines accessing extra-dimensions, but we expect our conclusions to apply to any extremely relativistic quanta which have access to the extradimensional bulk (thus using the term closed timelike curve (CTC) as being interchangeable to closed lightlike curve). Examples of such quanta are the graviton and the hypothetical gauge-singlet "sterile" neutrino.

We begin the CTC story by reviewing closed timelike curves in a prominent class of spacetimes including the Gödel- and Tipler-van-Stockum (GTvS) spacetimes in Section 2. In Section 3 we extend the discussion of possible CTCs to higher-dimensional spacetimes, and derive the general conditions on the metric which allow CTCs. We discuss the causality properties of asymmetrically warped 5-dimensional spacetimes in Section 4, and show that CTCs do not occur (unless spacetime has the topology of a flat torus). In contrast, we show in Section 5 that closed timelike curves can exist within a 6-dimensional generalization of asymmetrically warped spacetimes. We explicitly find a metric with two warped extra space dimensions which allows CTCs. In Section 6 we discuss the energy conditions for the 6D metric with CTCs. A brief discussion of the role that sterile (gauge-singlet) neutrinos may play in the communication with the past or future and a recapitulation and discussion complete this paper in Section 7.

\section{CTCs in Gödel and Tipler-van-Stockum spacetimes}

Before discussing the causality properties of asymmetrically warped spacetimes, it is instructive to review two prominent early examples of spacetimes implementing closed timelike 
curves. The Gödel metric describes a pressure-free perfect fluid with negative cosmological constant and rotating matter, and the Tipler-van-Stockum (TvS) spacetime is being generated by a rapidly rotating infinite cylinder. In both cases the metric can be written as

$$
d s^{2}=+g_{t t}(r) d t^{2}+2 g_{t \phi}(r) d t d \phi-g_{\phi \phi}(r) d \phi^{2}-g_{r r} d r^{2}-g_{z z} d z^{2}
$$

Here the $g_{\mu \nu}$ are complicated functions of the radial distance $r$ from the symmetry axis, and a parameter characterizing the angular velocity of the cylinder (not shown). The azimuthal coordinate $\phi$ assumes values on the interval $\phi \in[0,2 \pi\}$. Writing $g_{t t} d t^{2}+2 g_{t \phi} d t d \phi-g_{\phi \phi} d \phi^{2}$ as $\left(g_{t t}+\Omega^{2} g_{\phi \phi}\right) d t^{2}-g_{\phi \phi}(d \phi-\Omega d t)^{2}$, with $\Omega=g_{t \phi}(r) / g_{\phi \phi}(r)$, makes it clear that the Gödel and TvS metrics have an interpretation in terms of a rotating coordinate system with a radially-dependent angular speed $\Omega(r)$. The sign of the rotation is positive if $g_{t \phi}$ and $g_{\phi \phi}$ have the same relative sign, and negative if the relative sign is opposite.

To guarantee a local Minkowskian metric at every spacetime point, on and off the brane, we maintain the Lorentzian signature. The 4D metric has Lorentzian signature provided that $g_{4} \equiv \operatorname{Det}\left(g_{\mu \nu}\right)<0$. For $g_{r r} g_{z z}>0$ as in the Gödel and TvS (GTvS) metrics, this condition becomes

$$
\left(g_{t t} g_{\phi \phi}+g_{t \phi}^{2}\right)>0 .
$$

A dynamical approach to GTvS causality examines the purely azimuthal null-curve with $d s^{2}=0$. One gets

$$
\dot{\phi}_{ \pm}=\frac{g_{t \phi} \pm \sqrt{g_{t \phi}^{2}+g_{t t} g_{\phi \phi}}}{g_{\phi \phi}}
$$

where the \pm refers to co-rotating and counter-rotating lightlike signals. The coordinate time for a co-rotating path is

$$
\Delta T_{+}=\Delta \phi\left(\frac{g_{\phi \phi}}{g_{t \phi}+\sqrt{g_{t \phi}^{2}+g_{\phi \phi} g_{t t}}}\right) .
$$

As $g_{\phi \phi}$ goes from positive to negative, the light-cone tips such that the azimuthal closed path is traversed in negative time

$$
\Delta T_{+}=\frac{-2 \pi\left|g_{\phi \phi}\right|}{g_{t \phi}+\sqrt{g_{t \phi}^{2}+g_{\phi \phi} g_{t t}}} \quad\left[g_{\phi \phi}<0\right] .
$$

The quantum returns to its origin before it left, marking the existence of a CTC. 
Note that the Lorentzian signature is maintained even as $g_{\phi \phi}$ switches sign as long as the argument of the square-root, proportional to $-g_{4}$, remains positive definite. Note also that the Lorentzian signature is maintained as $g_{\phi \phi}$ switches to a negative value as long as $g_{t \phi}>\sqrt{-g_{\phi \phi} g_{t t}}$. In particular, $g_{t \phi}$ cannot be zero. In the following we will apply a similar argument to different scenarios of asymmetrically warped spacetimes.

For completeness, we give the analogous result for the counter-rotating light signal. The period of counter-rotation is

$$
\Delta T_{-}=\frac{2 \pi\left(g_{t \phi}+\sqrt{-g_{4}}\right)}{g_{t t}} .
$$

If $g_{t t}$ switches its sign at some $r_{0}$, then $\Delta T_{-}$becomes negative. However, such a sign change either produces singular behavior in $\Delta T_{-}$or requires a discontinuous behavior in $g_{t t}$. In the GTvS models, $g_{t t}$ does not change sign.

The clear discriminator of the arrows of time are the slopes of the local light-cone,

$$
s_{ \pm}(r)=\left(r \dot{\phi}_{ \pm}\right)^{-1}=\frac{1}{r} \frac{g_{\phi \phi}}{g_{t \phi} \pm \sqrt{-g_{4}}}=-\frac{1}{r} \frac{g_{t \phi} \mp \sqrt{-g}}{g_{t t}} .
$$

Notice that if $g_{\phi \phi}$ and $g_{t t}$ are positive, then regardless of the sign of $g_{t \phi}$, the light-cones (worldlines) remain in the first and second quadrants of the $(t, \phi)$ plane (as is the case of the Minkowski light-cone). Thus, for a backward flow of time, $g_{\phi \phi}$ (or, $\left.g_{t t}\right)$ must go through zero and become negative.

It is useful to consider the product of slopes

$$
s_{+}(r) s_{-}(r)=\frac{-1}{r^{2}} \frac{g_{\phi \phi}}{g_{t t}} .
$$

For time to move backwards one of the world lines defining the light-cone must move into the lower half of the $t-\phi$ plane. From (8) one can see that (i) this happens smoothly if $g_{\phi \phi}$ goes through zero; (ii) happpens discontinuously if $g_{t t}$ goes through zero; (iii) that a smooth change in the sign of $g_{t \phi}$ cannot move either slope through zero to the domain of negative time.

With the focus here on a smooth change of sign for $g_{\phi \phi}$, it is useful to examine the slopes at small $g_{\phi \phi}$. One finds

$$
s_{ \pm}\left(\text {leading order in } g_{\phi \phi}\right)=\left\{\begin{array}{c}
\frac{1}{2 r} \frac{g_{\phi \phi}}{g_{t \phi}} \\
-\frac{2}{r} \frac{g_{t \phi}}{g_{t t}}
\end{array}\right.
$$

It is clear that the slope $s_{+}$goes through zero with $g_{\phi \phi}$, leaving the first quadrant and moving into the fourth quadrant. With increasing $\phi$, time for the associated co-rotating 
world line runs backwards. On the other hand, the sign of $s_{-}$remains unchanged, and time for the associated counter-rotating world line continues to run forward. In the following we will apply similar arguments to different scenarios of asymmetrically warped spacetimes.

It is instructive to mention the visceral arguments against the relevance of the Gödel and TvS metrics. First of all, they are not asymptotically flat, and so presumably cannot occur within our Universe; rather, they must be our Universe, which contradicts observation. Secondly, the initial conditions from which they can evolve are either non-existent (Gödel) or sick (TvS). Furthermore, the TvS metric assumes an infinitely-long cylinder of matter, which is unphysical. On the positive side, literally, the Einstein equation endows $\rho=T_{0}^{0}=$ $\left(R_{0}^{0}-\frac{1}{2} R\right) / 8 \pi G_{N}$ (with the geometric RHS determined by the metric) with a positive value everywhere; there is no need for "exotic" $\rho<0$ matter. A further positive feature is the simplicity of finding the CTC by travel along the periodic variable $\phi$. We will later revisit these pluses and minuses with our results for asymmetrically warped spacetimes.

\section{Extending GTvS spacetimes to extra dimensions}

In this section we will model 6D spacetimes after the GTvS spacetimes. We label the two additional space dimensions $u$ and $v$, and we "warp" the bulk by letting the metric coefficients of the familiar spacetime coordinates $(t, \vec{x})$ depend on the bulk coordinates $u$ and $v$.

\subsection{A periodic path off the brane}

By construction, the causal properties of the metric depend only on the bulk coordinates $u$ and $v$. Therefore, we are led to consider first a path off the brane whose projection onto the brane is periodic. Such a path mimics closely that of GTvS. We begin with the 6D line element and metric

$$
d s^{2}=g_{t t}(u, v) d t^{2}+2 g_{t \phi}(u, v) d t(r d \phi)-g_{\phi \phi}(u, v)(r d \phi)^{2}-d r^{2}-d u^{2}-d v^{2}
$$

the brane coordinate $\theta$ is not needed, and so we have set it equal to $\pi / 2$. For this line element we have explicitly displayed the powers of $r$ so that all elements of $g_{\mu \nu}$ are dimensionless. The Lorentzian signature is maintained everywhere as long as $-g_{6} \equiv-\operatorname{Det}\left(g_{\mu \nu}\right)=\left(g_{t t} g_{\phi \phi}+\right.$ $\left.g_{t \phi}^{2}\right) r^{2}>0$ everywhere.

The algebra yielding the travel time for a periodic path in the hyperslice $(u, v)=\left(u_{1}, v_{1}\right)$ is little changed from the GTvS prototype. For fixed $r \equiv r_{1}$ we readily arrive at the travel 
time along a co-rotating path:

$$
\Delta T_{+}=r_{1} \Delta \phi\left(\frac{g_{\phi \phi}\left(u_{1}, v_{1}\right)}{g_{t \phi}\left(u_{1}, v_{1}\right)+\sqrt{-g_{6}\left(u_{1}, v_{1}\right)}}\right) .
$$

We take $\Delta \phi>0$ and $g_{t \phi}>0$. The transit time for the periodic co-rotating path is therefore

negative if $g_{\phi \phi}\left(u_{1}, v_{1}\right)$ is negative. Since the periodic path may be transited an arbitrary number of times, the finite time required for the lightlike quanta to travel from the brane at $(u, v)=(0,0)$ to the hyperslice in the bulk at $(u, v)=\left(u_{1}, v_{1}\right)$ and back can be neglected. Taking $\Delta \phi$ to be a multiple of $2 \pi$, one obtains a CTC.

We have little intuition for a globally-defined, differentially-rotating coordinate system. On the other hand, it may be possible to construct a coordinate system with rotation over a finite volume, e.g., by embedding a Kerr-like solution in the 6D space. However, such a construction, if possible at all, is a complication beyond the scope of this paper. We choose to leave the concept of a relative rotation between brane and bulk to future study, and instead consider next a related but different metric.

\subsection{A linear path off the brane}

We may replace the periodic coordinate of GTvS with the unbounded $x$ coordinate, and omit the $y$ and $z$ coordinates for brevity. Then one obtains

$$
d s^{2}=g_{t t}(u, v) d t^{2}+2 g_{t x}(u, v) d x d t-g_{x x}(u, v) d x^{2}-d u^{2}-d v^{2}
$$

(Notice in particular the sign convention on the coefficient of $d x^{2}$.)

The speed of light at any point will depend on $(u, v)$ through the metric elements. The restriction to Lorentzian signature implies that

$$
-g_{6} \equiv-\operatorname{Det}\left(g_{\mu \nu}\right)=g_{t t}(u, v) g_{x x}(u, v)+g_{t x}^{2}(u, v)>0
$$

World lines for lightlike travel (null lines) satisfy

$$
0=g_{t t}(u, v)+2 g_{t x}(u, v) \dot{x}-g_{x x}(u, v) \dot{x}^{2}-\dot{u}^{2}-\dot{v}^{2} .
$$

The solutions to (14) for the analogs of co-rotating and counter-rotating light speed at fixed $(u, v)$ are

$$
\dot{x}_{ \pm}=\frac{g_{t x}(u, v) \pm \sqrt{-g_{6}}}{g_{x x}(u, v)} .
$$

On the brane, $\dot{x}$ must equal $c=1$, so we again choose $g_{t t}(0,0)=g_{x x}(0,0)=1$ and $g_{t x}(0,0)=0$. 
Let us examine more closely the causal implications of Eq. (15). We assume that $g_{t t}$ is everywhere positive, so that (i) coordinate time $t$ is everywhere timelike, and (ii) no singularities are introduced in $s_{+} s_{-}$or in $g_{t t}$. As shown in Section (2), the sign of $g_{t x}$ does not influence the causal structure, and for definiteness we take it to be positive semidefinite. It is the sign of the metric element $g_{x x}$ that has smooth causal significance.

Similar to the causal analysis of the GTvS model of Section (2), we write the two slopes of the light-cone as

$$
s_{ \pm}(u, v)=\left(\dot{x}_{ \pm}\right)^{-1}=\frac{g_{x x}(u, v)}{g_{t x}(u, v) \pm \sqrt{g_{t x}^{2}(u, v)+g_{x x}(u, v) g_{t t}(u, v)}}
$$

From this, one readily gets for the slopes (given in Eq. (16))

$$
s_{+} s_{-}=\frac{-g_{x x}}{g_{t t}} .
$$

It is easily seen that when $g_{x x}, g_{t t}$, and $g_{t x}$ are all positive, the slopes are of opposite sign, and are connected to the Minkowski metric in the smooth limit $g_{t x} \rightarrow 0$. Thus, with $g_{t t}$ and $g_{t x}$ assumed positive, time flows in the usual manner if $g_{x x}$ is positive. Furthermore, with $g_{x x}>0$, we have $\operatorname{sign}\left(g_{t x} \pm \sqrt{-g_{6}}\right)= \pm$, so that from Eq. (15), one has $\dot{x}_{+}>0$, and $\dot{x}_{-}<0$. Thus, a positive $g_{x x}$ (as in the Lorentz metric) offers the standard situation with time flowing forward and velocity $\dot{x}$ having either sign.

On the other hand, if $g_{x x}$ is negative, then Eq. (17) shows that one light-cone slope has changed sign. The small $g_{x x}$ limit of the slopes

$$
s_{ \pm}\left(\text {leading order in } g_{x x}\right)=\left\{\begin{array}{r}
\frac{g_{x x}}{2 g_{t x}} \\
-\frac{2 g_{t x}}{g_{t t}}
\end{array}\right.
$$

reveals that it is the positive slope which has passed through zero to become negative, signifying a world line moving from the first quadrant, through the $x$-axis, into the fourth quadrant where times flows backwards for increasing $x$. With both slopes negative, one has that $\dot{x}_{ \pm}\left(g_{x x}<0\right)<0$. Thus, travel with increasing time is in the negative $x$ direction, while travel with decreasing time is in the positive $x$ direction. We summarize the causal properties of the metric (12) in Table 1.

The world line which we investigate is the following: the signal travels first from the brane at $(u, v)=(0,0)$ to the hyperslice at $\left(u_{1}, v_{1}\right)$, then from $\left(u_{1}, v_{1}\right)$ to the hyperslice at $\left(u_{2}, v_{2}\right)$, and finally back from $\left(u_{2}, v_{2}\right)$ to the point of origin $(0,0)$ on the brane (see Fig. 1). While on the $\left(u_{1}, v_{1}\right)$ hyperslice, the signal travels a distance $\Delta X$ in the positive $x$-direction 


\begin{tabular}{|c||c|c|}
\hline & $g_{x x}>0$ & $g_{x x}<0$ \\
\hline \hline$\Delta T>0$ & $\dot{x}_{+}>0$ & \\
& $(\Delta x>0)$ & \\
\cline { 2 - 3 } & $\dot{x}_{-}<0$ & $\dot{x}_{-}<0$ \\
& $(\Delta x<0)$ & $(\Delta x<0)$ \\
\hline$\Delta T<0$ & & $\dot{x}_{+}<0$ \\
& & $(\Delta x>0)$ \\
\hline
\end{tabular}

Table 1: Solution types for metric (12), and their casual properties. In particular, no solution exists for motion backwards in time along the negative- $x$ direction.

over a negative time $\Delta T_{1}=-\left|\Delta T_{1}\right|$. While on the $\left(u_{2}, v_{2}\right)$ hyperslice, the signal travels back an equal negative distance $-\Delta X$ in time $\Delta T_{2}$ to close the spatial projection of the worldline on the brane. To close the worldline on the brane, it is necessary that $T_{2}+T_{1}<0$. (But not equal to zero, as we allow for small positive travel times from the brane at $(u, v)=(0,0)$ to $\left(u_{1}, v_{1}\right)$, from $\left(u_{1}, v_{1}\right)$ to $\left(u_{2}, v_{2}\right)$, and back from $\left(u_{2}, v_{2}\right)$ to $(0,0)$.)

The transit time $\left(\Delta T_{1}\right)_{ \pm}$for light to travel a positive distance $\Delta X>0$ at constant $\left(u_{1}, v_{1}\right)$, as viewed from the brane, is

$$
\begin{aligned}
\left(\Delta T_{1}\right)_{ \pm} & =\int_{0}^{\Delta T_{1}} d t=\int_{0}^{\Delta X} d x \frac{g_{x x}\left(u_{1}, v_{1}\right)}{g_{t x}\left(u_{1}, v_{1}\right) \pm \sqrt{-g_{6}\left(u_{1}, v_{1}\right)}} \\
& =\Delta X\left(\frac{g_{x x}\left(u_{1}, v_{1}\right)}{g_{t x}\left(u_{1}, v_{1}\right) \pm \sqrt{-g_{6}\left(u_{1}, v_{1}\right)}}\right) .
\end{aligned}
$$

The integrations on $d t$ and $d x$ are trivial because the metric does not depend on the coordinate time $t$ or brane variable $x$. According to Eq. (13), the Lorentz signature is maintained as long as $g_{t x}^{2}>g_{t t}\left(-g_{x x}\right)$. We have shown that the world line for $x_{+}$lies below the $x$-axis when $g_{x x}<0$, and so we require $g_{x x}\left(u_{1}, v_{1}\right)<0$ in order to gain negative time $\Delta\left(T_{1}\right)_{+}$ during travel on the $\left(u_{1}, v_{1}\right)$ hyperslice. From here on, we will simply use the label $\Delta T_{1}$ for this negative $\Delta\left(T_{1}\right)_{+}$solution on the $\left(u_{1}, v_{1}\right)$ hyperslice:

$$
\Delta T_{1} \equiv \Delta\left(T_{1}\right)_{+}=\Delta X\left(\frac{g_{x x}\left(u_{1}, v_{1}\right)}{g_{t x}\left(u_{1}, v_{1}\right)+\sqrt{-g_{6}\left(u_{1}, v_{1}\right)}}\right) .
$$

To close the worldline, the lightlike signal must return from positive $\Delta X$ to the origin $x=0$ in a time $\Delta T_{2}$ less than or equal to $\left|\Delta T_{1}\right|$. If this were to occur in a negative time, then we would have $g_{x x}<0$ and $\dot{x}>0$. Table 1 shows that there is no solution of this type available. So the return path must take place in positive time, with $\dot{x}<0$. Reference 
again to Table 1 reveals that the return solution is $\dot{x}_{-}$. In principle, the $\dot{x}_{-}$solution on the $\left(u_{1}, v_{1}\right)$ hyperslice provides a return path. However, it is easy to show that the return time $\Delta T_{2}$ for this solution exceeds $\left|\Delta T_{1}\right|$ and so fails to close the world line. Thus, we must go to a second hyperslice at $\left(u_{2}, v_{2}\right)$. We have

$$
\Delta T_{2}=\left[\int_{\Delta X}^{0} d x=-\Delta X\right]\left(\frac{g_{x x}\left(u_{2}, v_{2}\right)}{g_{t x}\left(u_{2}, v_{2}\right)-\sqrt{-g_{6}\left(u_{2}, v_{2}\right)}}\right),
$$

with $g_{x x}\left(u_{2}, v_{2}\right)$ of either sign.

The necessary condition relating the outgoing and return paths of a CTC is that the sum $\Delta T_{2}+\Delta T_{1}$ be less than zero. Equivalently, the CTC conditions are that

$$
\frac{-g_{x x}\left(u_{2}, v_{2}\right)}{g_{t x}\left(u_{2}, v_{2}\right)-\sqrt{-g_{6}\left(u_{2}, v_{2}\right)}}+\frac{g_{x x}\left(u_{1}, v_{1}\right)}{g_{t x}\left(u_{1}, v_{1}\right)+\sqrt{-g_{6}\left(u_{1}, v_{1}\right)}}<0
$$

and that

$$
g_{x x}\left(u_{1}, v_{1}\right)<0
$$

(recall our sign convention (12) for $g_{x x}$ ). Here $\Delta T_{1}<0, \Delta T_{2}>0$ has been used. Note that (22a) is both necessary and sufficient, while (22b) is implied by (22a), assuming that the negative time $\Delta T_{1}$ is accumulated during the travel on the $\left(u_{1}, v_{1}\right)$ hyperslice. Thus (22b) is a necessary but not a sufficient condition. It is nevertheless a useful guide for our analysis of candidates for CTC spacetimes. The two transit times $\Delta T_{1}$ and $\Delta T_{2}$ can be made arbitrarily long, and so the short-time paths from the brane to the $\left(u_{1}, v_{1}\right)$ hyperslice, from $\left(u_{1}, v_{1}\right)$ to the $\left(u_{2}, v_{2}\right)$ hyperslice, and from $\left(u_{2}, v_{2}\right)$ back to the brane, can be neglected; if we can show the existence of metric elements on the $\left(u_{2}, v_{2}\right)$ and $\left(u_{1}, v_{1}\right)$ hyperslices satisfying the constraints of Eqs. (22), we will have demonstrated the existence of a closed worldline for lightlike quanta. Since $\Delta T_{1}$ and $\Delta T_{2}$ can be made arbitrarily long, finite mass effects of order $1 / \gamma^{2}$ may be neglected, and so the same conditions enable CTCs for extremely relativistic timelike quanta.

It is worth remarking that besides the necessity of the inequality $g_{x x}\left(u_{1}, v_{1}\right)<0$ to generate a negative time path, it is also necessary that $g_{t x}\left(u_{1}, v_{1}\right) \neq 0$. Without this latter condition, the Lorentz signature could not be maintained when $g_{x x}<0$, and indeed, the square root in the second term in (22a) would become imaginary.

Noting that $\Delta T_{1}$ is negative and $\Delta T_{2}$ positive, we have $\Delta T_{1} / \Delta X=-1 /\left|\dot{x}_{1}\right|$ and $\Delta T_{2} / \Delta X=+1 /\left|\dot{x}_{2}\right|$. Thus, we may also interpret Eqs. (22) to say that

$$
\frac{1}{\left|\dot{x}_{2}\right|}+\frac{-1}{\left|\dot{x}_{1}\right|}<0, \quad \text { i.e., }\left|\dot{x}_{2}\right|>\left|\dot{x}_{1}\right| \text {. }
$$


In words, the quantum must return to the brane at a greater speed than that with which it exited, in order for the projection of the worldline onto the time axis to close. Put another way, the slope of the world line below the $x$-axis, $s_{1}=\frac{-1}{\left|\dot{x}_{1}\right|}$, must exceed in magnitude the slope of the return path, $s_{2}=\frac{1}{\left|\dot{x}_{2}\right|}$.

At this point we can see that the return path, like the outgoing path, must occur off the brane. The outgoing path in Eq. (19) generates a negative time as perceived on the brane. This event is spacelike, superluminal, with the event $\left|\Delta T_{1}\right| / \Delta X$ outside the light-cone and therefore less than 1. The inverse velocity $\Delta T_{1} / \Delta X=-\left|\Delta T_{1}\right| / \Delta X$ is therefore greater than -1 . The return path, if on the brane, would be equal to $1 / c=1$, and the inequality in (22a) would be unfulfilled. Equivalently, we may note that a return path on the brane occurs at speed $\dot{x}_{2}=1$, whereas the superluminal outgoing path occurs at speed $\left|\dot{x}_{1}\right|>1$; Eq. (23) is thus unfulfilled.

Since Eqs. (22) are the necessary and sufficient condition for a CTC, any metric failing to satisfy the inequalities in (22) has no CTC. On the other hand, we have seen that the GTvS model contains a CTC in the $2+1$ dimensional space $(r, \phi ; t)$. Thus, we expect that CTCs will populate some metrics in $N+1$ spaces, for any $N>2$, as well. Indeed, Eq. (22) summarizes the straightforward recipe for constructing metrics with CTCs in spaces equal to or larger than $2+1$. One simply needs (i) a $g_{x x}$ that passes through zero as a function of another spatial coordinate " $u$ ", (ii) a nonzero $g_{t x}$ in the region of $u$ where $g_{x x}<0$, and (iii) a return path suitably arranged with nonzero values $g_{x x}$ and $g_{t x}$ in another coordinate region of $u$. The mathematical construction of such metrics is not in question. What may be debated is the physics motivation for such metrics. In the following sections we will develop a metric with CTCs, motivated by a popular concept in particle physics and gravitation, extra-dimensional "warped" spaces.

\section{Causality with one warped extra dimension}

We consider the five-dimensional asymmetrically-warped line element with a single extra dimension which we label as " $u$ ":

$$
d s^{2}=d t^{2}-\sum_{i} \alpha^{2}(u)\left(d x^{i}\right)^{2}-d u^{2}
$$

$i=1,2,3$, with our brane located at the $u=0$ submanifold. With no loss of generality, we may take $\alpha(u)$ to be positive.

Variants of this warped spacetime (24) can be generated by AdS-Schwarzschild or AdS- 
Reissner-Nordström black holes in the bulk [27, 28], and have been proposed as solutions to the cosmological horizon problem [21], and as a possible way around Weinberg's nogo theorem for the adjustment of the cosmological constant [28]. They also have been discussed in the context of the gravitational generation of cosmic acceleration [29], and infrared modification of gravity [30]. Very recently it has been shown that sterile neutrinos propagating in such a spacetime can account for the LSND neutrino oscillation evidence, without the problems faced by conventional four-dimensional four-neutrino scenarios [31].

The warped spacetime of (24) allows shortcut geodesics connecting spacelike-separated events on the brane if $|\alpha(u)|<|\alpha(0)|$ for any $u \neq 0$. However, the metric (24) exhibits a global time function $t$. Thus, taken by itself this spacetime is causally stable and does not allow for CTCs.

The failure of (24) to support a CTC can also be seen in our CTC equations (22). Since $g_{x x}=\alpha^{2}$ in (24) cannot be negative without violating the assumed Lorentzian signature, the CTC condition (22b) cannot be satisfied.

Given that the metric (24) does allow spacelike geodesics (as viewed from the brane), a boosted observer may see a negative time for the outgoing path. It is of pedagogical value to investigate (24) in the coordinates of this boosted observer. This effort will serve as a precursor for a successful construction of a metric with CTCs in six dimensions in the next section.

The metric in (24) is in Gaussian normal form with respect to $u$ (i.e., $g_{t u}=g_{x_{i} u}=$ $0)$, so the induced metric on each hypersurface with constant $u$ is simply given by the extra-dimensional metric evaluated on the hypersurface. These induced metrics are purely Minkowskian, albeit with a different constant limiting velocity $c(u)=\alpha^{-1}(u)$ on each hypersurface. This means that a Lorentz symmetry can be defined for each hypersurface, but each hypersurface's Lorentz symmetry will not hold on any other hypersurface, as we now discuss.

It is natural to choose $c(u=0)=1$ such that the induced metric on the brane is given by $d s_{\text {brane }}^{2}=d t^{2}-d x^{2}$. There then follows the usual Lorentz symmetry under the familiar transformations on our brane:

$$
x^{\prime}=\gamma(x-\beta t), \quad t^{\prime}=\gamma(t-\beta x), \quad u^{\prime}=u=0
$$

or equivalently, the inverse transformation

$$
x=\gamma\left(x^{\prime}+\beta t^{\prime}\right), \quad t=\gamma\left(t^{\prime}+\beta x^{\prime}\right),
$$


with the usual definition $\gamma=\left(1-\beta^{2}\right)^{-1 / 2}$. However, physics at $u \neq 0$ (in the "bulk") is not invariant under this transformation.

The complete metric in the boosted system is given by the tensor transformation law

$$
g_{\alpha \beta}^{\prime}=\frac{\partial x^{\mu}}{\partial x^{\prime \alpha}} \frac{\partial x^{\nu}}{\partial x^{\prime \beta}} g_{\mu \nu}
$$

where $g_{\mu \nu}=\operatorname{diag}\left(1,-\alpha^{2},-1\right)$ is the Gauss-normal metric of Eq. (24). Using Eq. (26), the resulting boosted metric is

$$
g_{\mu \nu}^{\prime}=\left(\begin{array}{ccr}
\gamma^{2}\left(1-\beta^{2} \alpha^{2}\right) & \gamma^{2} \beta\left(1-\alpha^{2}\right) & 0 \\
\gamma^{2} \beta\left(1-\alpha^{2}\right) & -\gamma^{2}\left(\alpha^{2}-\beta^{2}\right) & 0 \\
0 & 0 & -1
\end{array}\right) .
$$

Notice that only for $\alpha^{2}=1$ is the metric Lorentz invariant. Such is the case on our brane, but generally not the case on other hypersurfaces. On other hypersurfaces, Eq. (24) gives the limiting velocity seen by local inhabitants in the rest frame as $\alpha^{-1}\left(u_{j}\right) \equiv \alpha_{j}^{-1}$. However, this value is not invariant under Lorentz boosts defined on our brane.

At first glance the metric (28) seems to belong to the to the broad class of metrics (11), which includes the Gödel- and Tipler-van-Stockum (GTvS) spacetimes. After all, $g_{t x} \neq 0$ where $\alpha \neq 1$, i.e., off the brane. Moreover, $\operatorname{sign}\left(g_{x x}\right)$ is adjustable. However, a significant difference from the GTvS metric is that, in the case here of a boosted asymmetrically warped extra dimension, the variable $x$ is not periodic (unless our universe has the topology of a flat torus). It is thus required to construct an explicit return path to the spacetime point of origin. We will now show that it is not possible to construct a return path that arrives sufficiently quickly to close the curve.

The first way we show the absence of a CTC is to inject the metric elements of (28) into the CTC conditions (22). A boost does not alter the determinant of the metric, so $\sqrt{g_{5}}=|\alpha|$. With $g_{t x}=\gamma^{2} \beta\left(1-\alpha^{2}\right)$, and $g_{x x}=\gamma^{2}\left(\alpha^{2}-\beta^{2}\right)$, one finds that the CTC condition in Eq. (22a) is satisfied only if $\left|\alpha_{1}\right|<-\left|\alpha_{2}\right|$, which is a contradiction. So there is no CTC.

Secondly, we show explicitly that the physics failure arises from unavailability of any return path to close the curve. For our present 5D discussion, the path is that of Fig. 1 when one ignores the sixth dimension coordinate $v$. The signal leaves our brane at the spacetime point $O=(t=0, x=0, u=0)$ and arrives at $u=u_{1} \neq 0$, and then propagates on the hypersurface at $u_{1}$ for a travel time $t$ with the limiting velocity $\left(\alpha\left(u_{1}\right)\right)^{-1} \equiv \alpha_{1}^{-1}$. We will assume that $0<\alpha_{1}<1$, so that the travel speed in the bulk is superluminal relative to travel speed on our metric. At time $t$, the signal may reenter our brane. In the limit $u_{1} \ll \alpha_{1}^{-1} t$, which is always fulfilled for sufficiently large $t$, the reentry point on our brane 
is $B^{\mu} \approx\left(t, x=\alpha_{1}^{-1} t, u=0\right)$. Since the distance to the reentry point $B^{\mu}$ is spacelike (i.e. outside the brane's light-cone), it may be transformed to negative time by a boost on our brane. The boosted point $B^{\prime \mu}$ is obtained by using the transformation (25). The point $B^{\prime \mu}$ has coordinates

$$
x^{\prime}=\gamma t\left(\alpha_{1}^{-1}-\beta\right), \quad t^{\prime}=\gamma t\left(1-\beta \alpha_{1}^{-1}\right) .
$$

It is clear that for

$$
0<\alpha_{1}<\beta<1 \quad\left[\text { equivalent to } g_{x x}\left(u_{1}\right)<0\right]
$$

an observer in the boosted frame on our brane sees the signal arrive in time with $t^{\prime}<0$, i.e., before it was emitted. However, this result alone does not imply any conflict with causality. In particular, it does not necessarily imply that spacetime is blessed with CTCs. To close the timelike curve, one has to show that the time $t^{\prime}$ during which the signal traveled backwards in time, is sufficiently large to allow a return from the spacetime point $B^{\prime \mu}=\left(t^{\prime},\left(x=\alpha_{1}^{-1} t\right)^{\prime}, 0\right)$ on our brane to the spacetime point of origin, $O=O^{\prime}=(0,0,0)$. The speed required to close the lightlike curve of the signal, as seen by the boosted observer on the brane, is

$$
c_{\mathrm{req}}^{\prime}=\frac{\left(x=\alpha_{1}^{-1} t\right)^{\prime}}{\left|t^{\prime}\right|}=\frac{1-\beta \alpha_{1}}{\beta-\alpha_{1}},
$$

where the latter expression results from inputting Eq. (25). It is easy to show that the condition $0<\alpha_{1}<\beta<1$ implies that $c_{\text {req }}^{\prime}$ itself is superluminal. Thus there is no return path on our brane which leads to a CTC. To generate a CTC the signal has to traverse another path (say, at constant $u_{2}$ ) which has a limiting velocity satisfying $c_{\text {bulk }}^{\prime} \geq c_{\text {req }}^{\prime}$ in the $v$-frame.

Using the general expression for the metric in Eq. (28), the null line element for this hypersurface is, with $\alpha_{2} \equiv \alpha\left(u_{2}\right)$,

$$
0=d s^{\prime 2}=\gamma^{2}\left\{\left(1-\beta^{2} \alpha_{2}^{2}\right) d t^{\prime 2}+2 \beta\left(1-\alpha_{2}^{2}\right) d x^{\prime} d t^{\prime}-\left(\alpha_{2}^{2}-\beta^{2}\right) d x^{2}\right\}
$$

There results a quadratic equation for $c_{\text {bulk }}^{\prime}$. From Table 1 we see that the only possible solution is $\dot{x}_{-}$, given by Eq. (15) with $v$ set to zero and $-g_{6}$ replaced by $g_{5}=\alpha_{2}^{2}$. Thus we have

$$
c_{\mathrm{bulk}-}^{\prime} \equiv \dot{x}_{-}^{\prime}=\left(\frac{d x^{\prime}}{d t^{\prime}}\right)_{-}=\frac{\gamma^{2} \beta\left(1-\alpha_{2}^{2}\right)-\alpha_{2}}{\gamma^{2}\left(\beta^{2}-\alpha_{2}^{2}\right)}=\frac{1+\beta \alpha_{2}}{\beta+\alpha_{2}} .
$$

It is relatively easy to see that this result satisfies the inequality chain $\left|c_{\text {bulk- }}^{\prime}\right|<|1 / \beta|<$ $\left|c_{\text {req }}^{\prime}\right|$. (One way to see this inequality chain is to recognize that the RHS of (33) has the form of the velocity addition formula in Special Relativity, where the velocity sum is bounded by 
unity when the "velocities" $\beta$ and $\alpha_{2}$ are themselves bounded by unity.) Thus, the return path cannot be superluminal and thus cannot close the timelike curve, and there is no CTC.

So we conclude that in an asymmetrically warped space with only one extra dimension (24), as well as its boosted equivalent (28), no CTCs exist if space dimensions are nonperiodic. 5-dimensional brane universes with one asymmetrically warped extra dimension are causally stable. (The exception is the topology of a flat torus, which maps spacetime into the class of GTVS spacetimes of Eq. (11).) We are thus led to consider next a spacetime with two asymmetrically warped extra dimensions. There we will find that CTCs do exist. The lesson learned from the attempt to formulate a 5D metric having CTCs will provide intuitive input into the construction of the $6 \mathrm{D}$ metric.

\section{CTCs with two warped extra dimensions}

We now proceed by constructing a 6D metric exhibiting CTCs, which is a natural generalization of the metric (24). Let " $u$ " and " $v$ " label the two extra space dimensions. We assume that these dimensions have warp factors $\alpha(u)$ and $\eta(v)$, respectively.

In our attempt to construct an asymmetrically warped metric exhibiting CTCs in 5D, we found that the metric in Eq. (24) allowed a quantum to travel superluminally into the bulk. Being outside our light-cone, the worldline of this quantum could be boosted to negative time by a Lorentz transformation on the brane. However, we showed that a superluminal return path to the brane was required to close the worldline and there was no such path. This failure can be traced to the fact that the Lorentz transformation was just a coordinate change, and so provided a change of view, but no new physics. What is needed is a nonzero $g_{t x}$ that cannot be removed by a linear transformation among brane coordinates. Introducing the 6th dimension provides a solution, first because it allows a superluminal return path along the additional 6th dimension, and second because it allows $g_{t x}(u, v)$ to be "hard-wired" into the metric so that it is not removable by a linear coordinate transformation on the brane. (Recall that we learned in Section (3), via Eqs. (22) and the discussion just below these, that a nonzero $g_{t x}(u, v)$ is a necessary ingredient for the existence of CTCs.)

A natural 6D generalization of (24) can be realized by assuming that the metric for the $u$ - and $v$ dimensions exhibits the simple form in (24), but in different Lorentz frames. This assumption seems natural for any spacetime with two or more extra dimensions, since there is no preferred Lorentz frame for the bulk, from the viewpoint of the brane. In analogy to 
(24) it could be realized by assuming two AdS-Schwarzschild or AdS-Reissner-Nordström black holes being located in the $u$ and $v$ dimension and moving with a relative velocity. This choice also ensures superluminal travel to as well as from the brane, as well as a Minkowskian metric on the brane. To construct this 6-dimensional metric explicitly, let us denote by $\beta_{u v}$ the "relative velocity" between the two Lorentz frames in which the $u$ and $v$ dimensions assume the simple form (24), respectively. We incorporate the " $u$-frame" slice at $v=0$ by retaining the warp factor $\alpha(u)$ on the brane coordinate $d x$, and we incorporate the "v-frame" slice at $u=0$ by writing the boosted metric in Eq. (32) with the warp $\alpha(u)$ now replaced by $\eta(v)$. The resulting full 6-dimensional metric then has the form

$d s^{2}=\gamma_{u v}^{2}\left\{\left[1-\beta_{u v}^{2} \eta^{2}(v)\right] d t^{2}+2 \beta_{u v} \alpha(u)\left[1-\eta^{2}(v)\right] d x d t-\alpha^{2}(u)\left[\eta^{2}(v)-\beta_{u v}^{2}\right] d x^{2}\right\}-d u^{2}-d v^{2}$.

One easily finds that - Det $\equiv-g_{6}=\alpha^{2}(u) \eta^{2}(v)$. That this determinant is independent of $\beta_{u v}$ is consistent with the interpretation of $\beta_{u v}$ as a kind of boost parameter. Of special importance for the existence of the CTC is the off-diagonal metric element $g_{t x}$, which is nonzero for $\eta(v) \neq 1$ (i.e., off the brane), and the metric element $g_{x x}$ which is of indeterminate sign. As a consistency check on the metric, we note that for $u=v=0$, i.e., on the brane, Eq. (34) reduces to 4-dimensional Minkowski spacetime.

We now present two arguments, parallel to those given in Sec. (41) for the 5D case, but leading to the opposite conclusion, namely that the metric (34) does support CTCs.

The first argument establishing the existence of a CTC is to show that the metric elements in (34) can be chosen to satisfy the two CTC conditions of Eq. (22). Inputting the metric elements into (22) , one finds, after some algebra, that the conditions reduce to

$$
\frac{\alpha_{2}\left(\beta_{u v}+\eta_{2}\right)}{1+\beta_{u v} \eta_{2}}<\frac{\alpha_{1}\left(\beta_{u v}-\eta_{1}\right)}{1-\beta_{u v} \eta_{1}},
$$

and

$$
\eta_{1}<\beta_{u v}
$$

The new feature here, as opposed to the 5D metric, is the freedom to choose $\alpha_{1}$ and $\alpha_{2}$ to ensure that the CTC conditions are satisfied. We see that any pair $\left(\alpha_{1}, \alpha_{2}\right)$ will do, as long as they satisfy

$$
\frac{\alpha_{2}}{\alpha_{1}}<\left(\frac{\beta_{u v}-\eta_{1}}{1-\beta_{u v} \eta_{1}}\right)\left(\frac{1+\beta_{u v} \eta_{2}}{\beta_{u v}+\eta_{2}}\right) .
$$

This inequality can always be satisfied by an arbitrarily small choice for $\alpha_{2}$.

One simple and successful choice is to set $\alpha_{1}=1$ and $\eta_{2}=1$, i.e., to take the outgoing path on the $u=0$ hyperslice and the return path on the $v=0$ hyperslice (and the steps (i) 


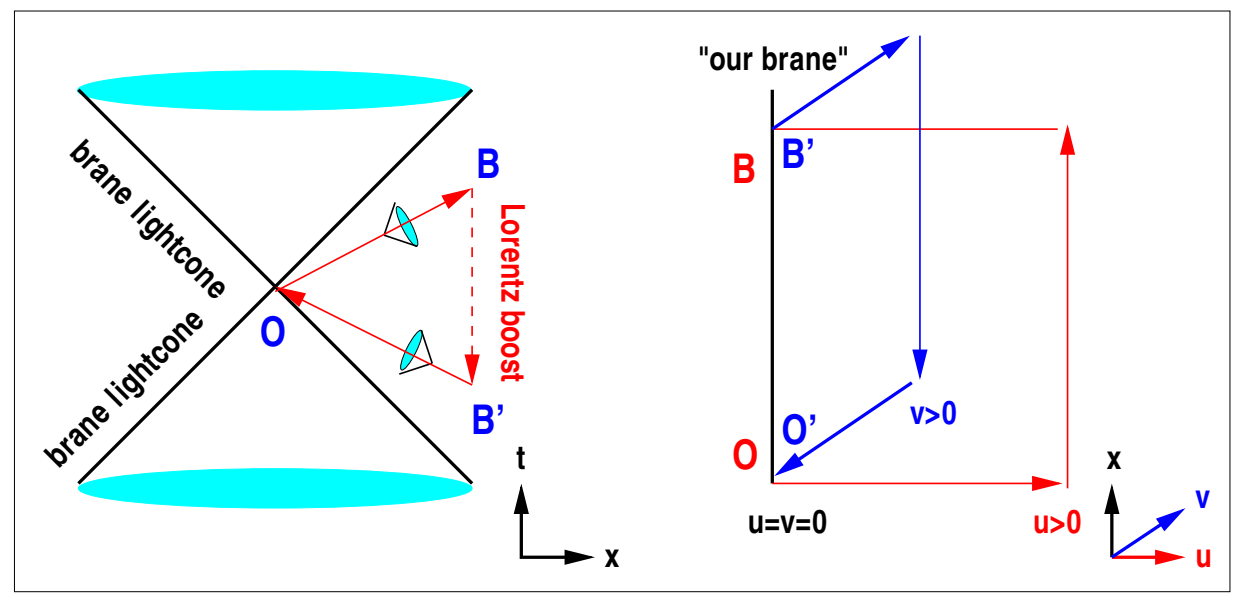

Figure 1: Closed timelike curve in an asymmetrically warped universe: (i) A signal takes a spacelike shortcut via a path of constant $u>0$ with $v=0$ from point $O$ to point $B$. (ii) A Lorentz boost transforms $B$ into $B^{\prime}$ with negative time coordinate. (iii) A return shortcut at constant $v>0$ with $u=0$ closes the timelike curve. The return path to the brane and the boost between steps (i) and (iii) is pedagogic rather than necessary, and an intermediate path in the bulk connecting the $u>0$ and $v>0$ hyperslices is possible. (Note: The tipped light-cones in the figure are symbolic rather than quantitative.)

and (iii) in Fig. 1 1 are interchanged). With these choices, (37) reduces to $\alpha_{2}<\left(\beta-\eta_{1}\right) /(1-$ $\left.\beta \eta_{1}\right)$. This is guaranteed to be satisfiable by (36). The choices $u_{1}=0$ and $v_{2}=0$ will lead to an explicit CTC. With $u=0$, Eq. (34) reduces to (32) with $\eta^{2}(v)$ replacing $\alpha^{2}(u)$ :

$$
\left.d s^{2}\right|_{u=0}=\gamma_{u v}^{2}\left\{\left[1-\beta_{u v}^{2} \eta^{2}(v)\right] d t^{2}+2 \beta_{u v}\left[1-\eta^{2}(v)\right] d x d t-\left[\eta^{2}(v)-\beta_{u v}^{2}\right] d x^{2}\right\}-d v^{2} .
$$

Thus we see explicitly that choosing $\eta_{1}<\beta_{u v}$ on the $u=0$ hyperslice sets $g_{x x}<0$, so that our outgoing path necessarily accumulates negative time (original frame in Table 2). On the return path, we set $v=0$. Then the $6 \mathrm{D}$ metric of Eq. (34) reduces to (24), repeated here:

$$
\left.d s^{2}\right|_{v=0}=d t^{2}-\alpha^{2}(u) d x^{2}-d u^{2} ;
$$

It is clear that this return path can be made arbitrarily brief by choosing $\alpha_{2}$ arbitrarily small. The CTC is revealed.

We note that when the metric (34) is transformed into the $v$-frame by a Lorentz transformation on the brane with $\beta=-\beta_{u v}$, then the metric along the $v$-dimension assumes the simple form of (39) (with obvious replacements) and the metric along the $u$-dimension becomes non-diagonal ( $v$-frame in Table 2). 


\begin{tabular}{|c|c|c|}
\hline & original $u$-frame & (un)-boosted $v$-frame \\
\hline \hline$u \neq 0$ & forward in time & backwards in time \\
\hline$v \neq 0$ & backwards in time & forward in time \\
\hline CTC & & \\
& &
\end{tabular}

Table 2: Running of time in the original frame, where the metric assumes the form (34), and in the frame where the metric is boosted into the $v$-frame with $\beta=-\beta_{u v}$.

To summarize this section, we have identified a CTC beginning and ending on our brane and superluminally transiting two paths parallel to our brane but in the asymmetrically warped $u$ - and $v$-dimensions. The physics that enables the CTC is the breaking of global Lorentz invariance away from the brane.

\section{Stress-energy tensor and energy conditions}

As a check on the consistency of the picture, we should diagnose the stress-energy tensor which sources the extra-dimensional metric, for any pathologies. In particular, we will be interested in the resulting matter distributions on and off the brane. Thus, our task is to calculate the Einstein tensor

$$
G_{\mu \nu}=R_{\mu \nu}-\frac{1}{2} g_{\mu \nu} R
$$

from the spacetime metric of Eq. (34), and then to obtain the stress-energy tensor $T_{\mu \nu}$ via the Einstein equation

$$
T_{\mu \nu}=\frac{1}{8 \pi \mathrm{G}_{\mathrm{N}}} G_{\mu \nu} .
$$

Consequently, we proceed to evaluate $T_{\mu \nu}=\left(8 \pi G_{\mathrm{N}}\right)^{-1} G_{\mu \nu}$ with no preconceptions as to its form. We note that in general, $T_{\mu \nu}$ contains contributions from matter, fields, and cosmological constant on and off the brane, and from brane tension on the brane.

Instead of complicated analytic expressions for $T_{\mu \nu}$, we present some visual output [32] of the Einstein tensor versus $u$, on the $v=0$ slice. We do so for warp factors $\alpha(u)$ and $\eta(v)$ chosen to satisfy energy conditions discussed below. An analogous figure is the Einstein tensor versus $v$, on the $u=0$ slice. However, this Einstein tensor has off-diagonal elements, 


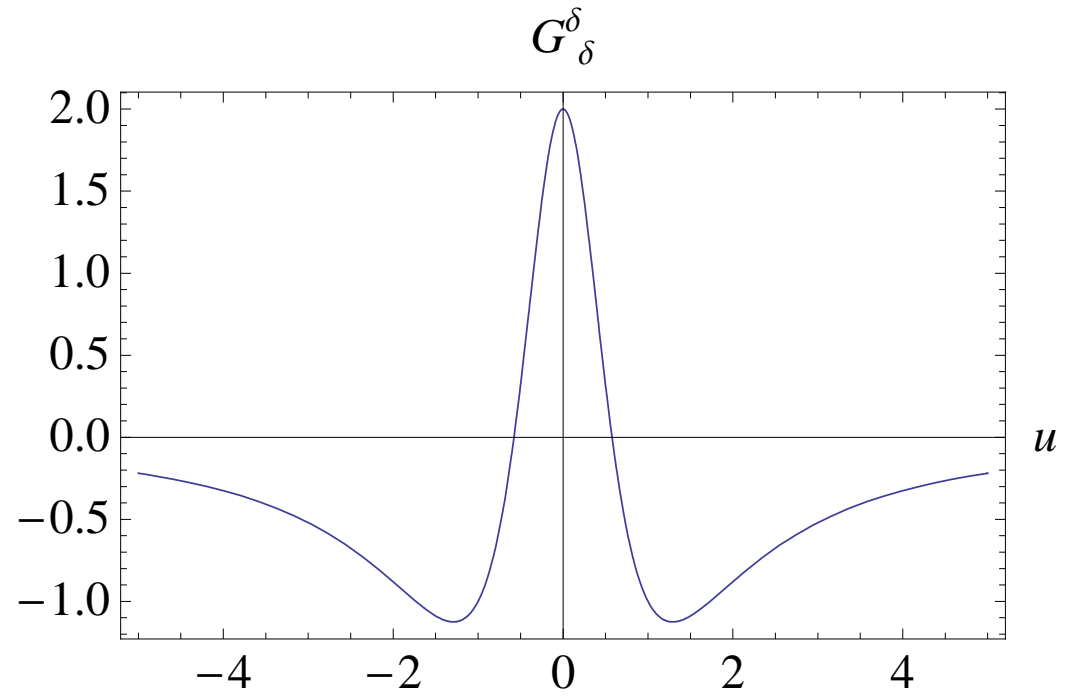

Figure 2: Nonzero elements of the Einstein tensor $G^{\mu}{ }_{\nu}$ (in arbitrary units): $G^{\delta}{ }_{\delta} \equiv G_{0}^{0}=$ $G_{y}^{y}=G_{z}^{z}=G_{v}^{v}$, on the $v=0$ slice, as a function of $u$. Assumed are warp factors $\alpha(u)=1 /\left(u^{2}+c^{2}\right)$ and $\eta(v)=1 /\left(v^{4}+c^{2}\right)$, with $c=1$. We find that the weak and dominant energy conditions are violated in the bulk, while all energy conditions with the exception of the SEC are satisfied on the brane.

which increases the number of figures. Furthermore, it offers us no additional enlightenment, so we do not show this Einstein tensor.

There is considerable theoretical prejudice that stable Einstein tensors should satisfy certain "energy conditions" relating energy density $\rho$ and directional pressures $p^{j}$. The null, weak, strong and dominant energy conditions state that

$$
\begin{aligned}
\text { NEC } & : \quad \rho+p^{j} \geq 0, \quad \forall j . \\
\text { WEC }: & \rho \geq 0 ; \quad \text { and } \quad \rho+p^{j} \geq 0, \quad \forall j . \\
\text { SEC }: & : p^{j} \geq 0, \quad \forall j ; \quad \text { and } \rho+\sum_{j} p^{j} \geq 0 . \\
\text { DEC } & : \quad \rho \geq 0 ; \quad \text { and } \quad p^{j} \in[\rho,-\rho], \quad \forall j .
\end{aligned}
$$

For the purpose of definiteness in the identification of $\rho$ and $p^{j}$, we assume the anisotropic fluid relations

$$
T^{\mu \nu}=-p g^{\mu \nu}+(\rho+p) U^{\mu} U^{\nu},
$$

with $u^{\mu}=(1, \overrightarrow{0})$ being the net four velocity of the fluid. The usual approach is to work with one raised and one lowered index to express the stress-energy in terms of metric $g_{\mu \nu}$ rather 
than inverse metric $g^{\mu \nu}$. One has

$$
T_{\nu}^{\mu}=-p \delta_{\nu}^{\mu}+(\rho+p) g_{\nu \alpha} U^{\mu} U^{\alpha}
$$

Then, with a diagonal metric with $g_{t t}=1$ (Gaussian-normal coordinates), one obtains for the nonzero elements of $T_{\mu}^{\mu}$,

$$
\rho=T_{0}^{0} \quad \text { and } \quad p^{j}=-T_{j}^{j} .
$$

These are the relations appropriate for the $v=0$ slice of our metric, since one sees in Eq. (39) that the $v=0$ metric is manifestly diagonal with $g_{t t}=1$.

It is not difficult to find a functional form for the warp factors $\alpha$ and $\eta$ which conserves some of the energy conditions, at least on the brane. One such example is given by $\alpha(u)=$ $1 /\left(u^{2}+c^{2}\right)$ and $\eta(v)=1 /\left(v^{4}+c^{2}\right)$. For this case the elements of the Einstein tensor on the $v=0$ slice are shown as a function of $u$ in Fig. 2. The null, weak and dominant energy

conditions are conserved on the brane, while the strong energy condition is violated both on the brane and in the bulk.

The negative energy density that afflicts many wormhole and CTC solutions in four dimensions is avoided on the brane in the example for an extra-dimensional CTC presented here. However, $\rho$ becomes negative as one moves away from the brane into the bulk, so that the WEC and DEC are violated off the brane, while the NEC remains satisfied. We have successfully constructed a metric exhibiting CTCs in an extra-dimensional spacetime by "moving" the negative energy density from the brane to the bulk. One might even speculate that the negative energy density in the bulk is related to the compactification of the extra dimensions, or possibly to the repulsion of Standard matter from the bulk.

One also sees in Fig. (2) that $G_{y}^{y}=G_{z}^{z}=G_{v}^{v}$ are equal to $G_{0}^{0}$ on the $v=0$ slice. This equality amounts to a dark energy or cosmological constant equation of state for the $y$-, $t$-, and $v$-directed pressures, namely, $w^{j} \equiv p^{j} / \rho=-1$. There may be some intriguing physics underlying this result.

\section{Discussion and Conclusion}

We have derived the general conditions on metric elements which allow spacetimes to contain closed timelike curves (CTCs). Then, we have demonstrated the existence of CTCs for a rather generic spacetime with two asymmetrically warped extra dimensions. In addition, we have found particular warp factors for the metric which yield positive energy density 
on the brane. However, negative energy density is not completely banished, as it does appear in the bulk. Since one cannot observe the bulk energy density, we may at least say that negative energy density is banished from sight. It is also possible that an anthropic argument applies here: Life may evolve only where energy density is positive. Then lifeless bulk regions of negative energy density can communicate their existence to living beings only via geometry, perhaps mediated by the exchange of gravitons or appropriately named, "sterile" neutrinos.

It should be stressed that realistic graviton or bulk fermion signals, rather than following restricted bulk trajectories with constant $u$ or $v$ as constructed here, will instead propagate on the path of least action to minimize the travel time. Since the effectively superluminal velocities in our constructed example produced a CTC, we expect that a truly geodesic signal will also generate a CTC. In this case the causal structure of extra dimensions may be studied with sterile neutrino beams by utilizing resonant conversion of active neutrinos via matter effects into sterile neutrinos and back. We note that the model presented herein is complete in that the geodesic equations of motion are derivable from the metric in Eq. (34). We have not investigated the geodesic equations in this work.

A thorough discussion of whether CTCs in the observable universe are hidden behind chronology horizons where the stress-energy tensor diverges (one may consult the discussion in [33, 34]), is beyond the scope of this work. We have confined ourselves to the pragmatic attitude that even if chronology were protected by some mechanism operative near the chronology horizon, it remains a highly rewarding effort to study the physics near this horizon. The CTC we have constructed is particularly interesting in this respect, since it could be available to gauge-singlet particles which have previously been hypothesized to propagate in the extra-dimensional bulk 2 .

\section{Acknowledgments}

We thank the referee, whose constructive criticism improved the paper considerably. We also thank Luis Anchordoqui, Roman Buniy, Cliff Burgess, Daniel Chung, Joshua Erlich, John Friedman, Alan Guth, Daniel Hobbs, Hideki Ishihara, Nick Kaiser, Thomas W. Kephart, Jeff Kuhn, John G. Learned, Ralf Lehnert, Kirill Melnikov, Octavian Micu, Alexander Mitov, Graham Shore, Xerxes Tata, Matthew Weippert, and T.-C. Yuan for many critical

\footnotetext{
${ }^{2} \mathrm{~A}$ realistic description of such particles taking shortcuts in extra dimensions would require a quantum field theoretic treatment similar to the one preformed in [35.
} 
comments and useful discussions. We thank the Tokkuri Tei establishment and especially Kazu Mitake for providing a stimulating environment in which to further this paper. This work was supported in part by US DOE under the grants DE-FG02-04ER41291 and DEFG05-85ER40226. TJW thanks the Alexander von Humboldt Foundation for support with a Senior Research Award. HP and TJW thank the Kavli Institute for Theoretical Physics China for kind support and hospitality during the last stages of this work.

\section{References}

[1] M. S. Morris and K. S. Thorne, Wormholes In Spacetime And Their Use For Interstellar Travel: A Tool For Teaching General Relativity, Am. J. Phys. 56, 395 (1988); M. S. Morris, K. S. Thorne and U. Yurtsever, Wormholes, Time Machines, And The Weak Energy Condition, Phys. Rev. Lett. 61, 1446 (1988).

[2] K. Gödel, An Example Of A New Type Of Cosmological Solutions Of Einstein's Field Equations Of Graviation, Rev. Mod. Phys. 21, 447 (1949).

[3] W. J. van Stockum, Gravitational field of a distribution of particles rotating around an axis of symmetry, Proc. R. Soc. Edin. 57 135-154 (1937).

[4] F. J. Tipler, Rotating cylinders and the possibility of global causality violation, Phys. Rev. D9, 2203-2206 (1974).

[5] J. R. I. Gott, Closed Timelike Curves Produced By Pairs Of Moving Cosmic Strings: Exact Solutions, Phys. Rev. Lett. 66, 1126 (1991).

[6] S. Deser, R. Jackiw and G. 't Hooft, Physical cosmic strings do not generate closed timelike curves, Phys. Rev. Lett. 68, 267 (1992); S. Deser and R. Jackiw, Time travel?, Comments Nucl. Part. Phys. 20, 337 (1992) |arXiv:hep-th/9206094|; G. M. Shore, Constructing time machines, Int. J. Mod. Phys. A 18, 4169 (2003) arXiv:gr-qc/0210048;

G. M. Shore, Causality and superluminal light, arXiv:gr-qc/0302116.

[7] J. A. Wheeler, Geons, Phys. Rev. 97 511-536 (1962); J. A. Wheeler, On the nature of quantum geometrodynamics, Ann. Phys. (NY) 2 511-536 (1962); J. A. Wheeler, Geons, Phys. Rev. 97 511-536 (1962).

[8] S. W. Hawking, G. F. R. Ellis, The Large Scale Structure of Spacetime, (Cambridge University Press) 1973. 
[9] M. Alcubierre, The warp drive: hyper-fast travel within general relativity, Class. Quant. Grav. 11, L73 (1994) arXiv:gr-qc/0009013; A. E. Everett, Warp drive and causality, Phys. Rev. D 53, 7365 (1996).

[10] A. Ori, A new time-machine model with compact vacuum core, arXiv:gr-qc/0503077.

[11] S. W. Kim and K. P. Thorne, Do vacuum fluctuations prevent the creation of closed timelike curves?, Phys. Rev. D 43, 3929 (1991); B. S. Kay, M. J. Radzikowski and R. M. Wald, Quantum field theory on spacetimes with a compactly generated Cauchy horizon, Commun. Math. Phys. 183, 533 (1997) arXiv:gr-qc/9603012 ; R. V. Buniy and S. D. H. Hsu, Semi-classical wormholes and time machines are unstable, arXiv:hep-th/0504003.

[12] S. W. Hawking, The Chronology protection conjecture, Phys. Rev. D 46, 603 (1992).

[13] M. Visser, Lorentzian Wormholes - from Einstein to Hawking, (Springer, New York) 1996; M. Visser, The quantum physics of chronology protection, Proc. Cambridge 2002: The future of theoretical physics and cosmology, arXiv:gr-qc/0204022.

[14] D. Deutsch, Quantum mechanics near closed timelike lines, Phys. Rev. D 44, 3197 (1991).

[15] L. Dyson, Chronology protection in string theory, JHEP 0403, 024 (2004) arXiv:hep-th/0302052; M. M. Caldarelli, D. Klemm and P. J. Silva, Chronology protection in anti-de Sitter, arXiv:hep-th/0411203.

[16] N. Arkani-Hamed, S. Dimopoulos and G. R. Dvali, The hierarchy problem and new dimensions at a millimeter, Phys. Lett. B 429, 263 (1998) |arXiv:hep-ph/9803315]; I. Antoniadis, N. Arkani-Hamed, S. Dimopoulos and G. R. Dvali, New dimensions at a millimeter to a Fermi and superstrings at a TeV, Phys. Lett. B 436, 257 (1998) arXiv:hep-ph/9804398; N. Arkani-Hamed, S. Dimopoulos and G. R. Dvali, Phenomenology, astrophysics and cosmology of theories with sub-millimeter dimensions and TeV scale quantum gravity, Phys. Rev. D 59, 086004 (1999) arXiv:hep-ph/9807344.

[17] L. Randall and R. Sundrum, A large mass hierarchy from a small extra dimension, Phys. Rev. Lett. 83, 3370 (1999) arXiv:hep-ph/9905221]; L. Randall and R. Sundrum, An alternative to compactification, Phys. Rev. Lett. 83, 4690 (1999) arXiv:hep-th/9906064; N. Arkani-Hamed, S. Dimopoulos, G. R. Dvali and 
N. Kaloper, Infinitely large new dimensions, Phys. Rev. Lett. 84, 586 (2000) arXiv:hep-th/9907209.

[18] J. L. Hewett and J. March-Russell, pages 1056-1064 of the Review of Particle Physics, Phys. Lett. B592 1 (2004); F. Feruglio, Eur. Phys. J. C 33, S114 (2004) arXiv:hep-ph/0401033.

[19] N. Arkani-Hamed, S. Dimopoulos, G. R. Dvali and J. March-Russell, Neutrino masses from large extra dimensions, Phys. Rev. D 65, 024032 (2002) arXiv:hep-ph/9811448; K. R. Dienes, E. Dudas and T. Gherghetta, Light neutrinos without heavy mass scales: A higher-dimensional seesaw mechanism, Nucl. Phys. B 557, 25 (1999) arXiv:hep-ph/9811428]; Y. Grossman and M. Neubert, Neutrino masses and mixings in non-factorizable geometry, Phys. Lett. B 474, 361 (2000) |arXiv:hep-ph/9912408; S. J. Huber and Q. Shafi, Majorana neutrinos in a warped 5D standard model, Phys. Lett. B 544, 295 (2002) arXiv:hep-ph/0205327); G. Bhattacharyya, H. V. KlapdorKleingrothaus, H. Päs and A. Pilaftsis, Neutrinoless double beta decay from singlet neutrinos in extra dimensions, Phys. Rev. D 67, 113001 (2003) |arXiv:hep-ph/0212169|; G. Bhattacharyya, H. V. Klapdor-Kleingrothaus, H. Päs and A. Pilaftsis, Double beta decay and the extra-dimensional seesaw mechanism, arXiv:hep-ph/0402071.

[20] G. Kaelbermann, Communication through an extra dimension, Int. J. Mod. Phys. A 15, 3197 (2000) arXiv:gr-qc/9910063|. G. Kaelbermann and H. Halevi, Nearness through an extra dimension, arXiv:gr-qc/9810083.

[21] D. J. H. Chung and K. Freese, Cosmological challenges in theories with extra dimensions and remarks on the horizon problem, Phys. Rev. D 61, 023511 (2000) arXiv:hep-ph/9906542]; D. J. H. Chung and K. Freese, Can geodesics in extra dimensions solve the cosmological horizon problem?, Phys. Rev. D 62, 063513 (2000) arXiv:hep-ph/9910235.

[22] D. Hochberg and T. W. Kephart, Can semiclassical wormholes solve the cosmological horizon problem?, Gen. Rel. Grav. 26, 219 (1994).

[23] H. Ishihara, Causality of the brane universe, Phys. Rev. Lett. 86, 381 (2001) arXiv:gr-qc/0007070.

[24] R. R. Caldwell and D. Langlois, Shortcuts in the fifth dimension, Phys. Lett. B 511, 129 (2001) arXiv:gr-qc/0103070. 
[25] H. Stoica, Comment on $4 D$ Lorentz invariance violations in the brane-world, JHEP 0207, 060 (2002) arXiv:hep-th/0112020.

[26] E. Abdalla, A. G. Casali and B. Cuadros-Melgar, Shortcuts in Cosmological Branes, Int. J. Theor. Phys. 43, 801 (2004) arXiv:hep-th/0501076].

[27] D. J. H. Chung, E. W. Kolb and A. Riotto, Extra dimensions present a new flatness problem, Phys. Rev. D 65, 083516 (2002) [arXiv:hep-ph/0008126].

[28] C. Csaki, J. Erlich and C. Grojean, Gravitational Lorentz violations and adjustment of the cosmological constant in asymmetrically warped spacetimes, Nucl. Phys. B 604, 312 (2001) arXiv:hep-th/0012143.

[29] A. Padilla, Cosmic acceleration from asymmetric branes, Class. Quant. Grav. 22, 681 (2005) arXiv:hep-th/0406157.

[30] A. Padilla, Infra-red modification of gravity from asymmetric branes, Class. Quant. Grav. 22, 1087 (2005) arXiv:hep-th/0410033].

[31] H. Päs, S. Pakvasa and T. J. Weiler, Sterile - active neutrino oscillations and shortcuts in the extra dimension, Phys. Rev. D 72, 095017 (2005) arXiv:hep-ph/0504096].

[32] To generate the Einstein tensor from the input metric, we have used the Mathematic notebook provided by J. B. Hartle, L. Parker http://www.physics.ucsb.edu/ gravitybook/math/curvature.nb

[33] W. F. Kao and C. Soo, A note on 4-dimensional traversable wormholes and energy conditions in higher dimensions, arXiv:gr-qc/0306004.

[34] P. F. Gonzalez-Diaz, Misner-brane cosmology, Phys. Lett. B 486, 158 (2000) arXiv:gr-qc/0004078.

[35] C. P. Burgess, J. Cline, E. Filotas, J. Matias and G. D. Moore, Loop-generated bounds on changes to the graviton dispersion relation, JHEP 0203, 043 (2002) arXiv:hep-ph/0201082]. 